\title{
Simvastatin enhances oxidized-low density lipoprotein-induced macrophage autophagy and attenuates lipid aggregation
}

\author{
BAOJUN HUANG ${ }^{1 *}$, MENGXING JIN $^{1 *}$, HAI YAN $^{1}$, YANWEI CHENG ${ }^{1}$, \\ DAKE HUANG $^{2}$, SONGCHENG YING ${ }^{1}$ and LINJIE ZHANG ${ }^{1}$ \\ ${ }^{1}$ Department of Immunology; ${ }^{2}$ Comprehensive Laboratory, School of Basic Medical Sciences, \\ Anhui Medical University, Hefei, Anhui 230032, P.R. China
}

Received March 31, 2014; Accepted October 2, 2014

DOI: $10.3892 / \mathrm{mmr} .2014 .2790$

\begin{abstract}
Macrophage autophagy exerts a protective effect in advanced atherosclerosis. It has previously been reported that oxidized low-density lipoprotein (ox-LDL) induces autophagy in endothelial cells, and simvastatin enhances autophagy in coronary arterial myocytes. However, it is currently unknown whether ox-LDL induces autophagy in macrophages, or whether simvastatin affects macrophage autophagy in atherosclerosis. The present study demonstrated that ox-LDL induced lipid accumulation in the J774A.1 macrophage cell line, in a dose-dependent manner, as determined by oil red $\mathrm{O}$ staining. Ox-LDL also induced autophagy in the J774A.1 cells, by converting microtubule-associated protein 1 light chain 3 (LC3) I to LC3 II, which is a well-known autophagy marker. Notably, treatment of the cells with simvastatin elevated ox-LDL-induced macrophage autophagy, this was detected through the conversion of LC3 I to LC3 II and the increased expression of Beclin1, another autophagy marker. Furthermore, it was shown that stimulation with ox-LDL led to the redistribution of green fluorescent protein (GFP)-LC3 from diffusion distribution, to the formation of puncta in the J774A.1 cells. Simvastatin promoted the ox-LDL-induced formation of GFP-LC3 puncta, as detected by confocal laser scanning microscopy. Simvastatin was also shown to inhibit ox-LDL-induced cholesterol accumulation in the J774A.1 cells, as observed by oil red $\mathrm{O}$ staining and CHOD-PAP assay. These results suggest that simvastatin may enhance ox-LDL-induced macrophage autophagy and attenuate lipid aggregation.
\end{abstract}

Correspondence to: Dr Songcheng Ying or Dr Linjie Zhang, Department of Immunology, School of Basic Medical Sciences, Anhui Medical University, .81 Meishan Road, Hefei, Anhui 230032, P.R. China

E-mail: yingsc@ahmu.edu.cn

E-mail: zlj33@ahmu.edu.cn

*Contributed equally

Key words: simvastatin, oxidized-low density lipoprotein, macrophage, autophagy, cholesterol

\section{Introduction}

Activation, dysfunction and structural alterations of the arterial endothelium results in the subendothelial retention of oxidized low-density lipoprotein (ox-LDL) from the plasma, thereby promoting the development and progression of atherosclerosis (1). Activated endothelial cells secrete chemokines, and subsequently express adhesion molecules, which may facilitate the recruitment of leukocytes, from the blood into the arterial wall (2). Of the leukocytes, monocytes are a critical cell type that contribute to the formation of atherosclerotic plaques. In the intima, migrated monocytes differentiate into macrophages, which engulf ox-LDL to form cholesterol-laden foam cells (3).

Autophagy is a conserved catabolic process in which cytoplasmic material is delivered to the lysosomal machinery for degradation and recycling. It is well-known that autophagy occurs in advanced atherosclerotic plaques (4-6), and that macrophage autophagy exerts a protective effect in advanced atherosclerosis (7). Previous studies have shown that ox-LDL may induce autophagy in endothelial cells, leading to the degradation of ox-LDL through autolysosomes $(8,9)$. However, little is currently known about whether ox-LDL may induce autophagy in macrophages.

Statins are inhibitors of 3-hydroxy-3-methylglutaryl-coenzyme A (HMG-CoA) reductase. Statins impede cholesterol biosynthesis, therefore reducing blood cholesterol levels. As well as lowering lipid levels, statins have been demonstrated to possess pleiotropic effects, including anti-inflammatory and immunomodulatory properties $(10,11)$. Simvastatin is a widely used statin, that exhibits lipid and non-lipid effects $(12,13)$. Recently, simvastatin was shown to enhance autophagy in coronary arterial myocytes, through inhibition of the Rac1-mammalian target of rapamycin (mTOR) signaling pathway (14). However, the effects of simvastatin on macrophage autophagy remain unclear.

The present study examined whether ox-LDL may induce autophagy in macrophages, and explored the role of simvastatin in ox-LDL-induced macrophage autophagy and lipid accumulation.

\section{Materials and methods}

Cell culture and reagents. The J774A.1 murine macrophage cell line was purchased from the China Center for Type 
Culture Collection (Wuhan, China). The cells were maintained in RPMI-1640 medium (Thermo Fisher Scientific, Co., Waltham, MA, USA), supplemented with $10 \%$ fetal calf serum (Zhejiang Tianhang Biological Technology Co., Ltd., Hangzhou, China), at $37^{\circ} \mathrm{C}$ in an atmosphere containing $5 \%$ $\mathrm{CO}_{2}$. Ox-LDL was purchased from Yiyuan Biotechnology Co., Ltd (Guangzhou, China). Simvastatin was purchased from Tokyo Chemical Industry Co., Ltd (Shanghai, China). Oil Red O dye and Total Cholesterol Detection kit were purchased from Nanjing Jiancheng Biotechnology Institute Co., Ltd (Nanjing, China). Lipofectamine ${ }^{\circledR} 2000$ was obtained from Life Technologies (Grand Island, NY, USA). Rabbit anti-microtubule-associated protein 1 light chain 3(LC3) and rabbit anti-Beclin1 antibodies were purchased from Cell Signaling Technology, Inc. (Danvers, MA, USA). Horseradish peroxidase (HRP)-labeled goat anti-rabbit Immunoglobulin $G$ was purchased from Beijing ZSGB-Biotechnology, Co. (Beijing, China). The Bicinchoninic Acid (BCL) Protein Assay kit was obtained from Beijing YPH-Bio Company (Beijing, China), and the Enhanced Chemiluminescence (ECL) Detection system was purchased from Pierce Biotechnology, Inc. (Rockville, MD, USA).

Establishment and detection of macrophage foam cell model. The J774A.1 cells were seeded into 6 -well plates $\left(5 \times 10^{5}\right.$ cells per well) and grown to 50-60\% confluency. A total of $0,25,50$ or $100 \mu \mathrm{g} / \mathrm{ml}$ ox-LDL was added to the cell supernatants, and the cells were incubated for $24 \mathrm{~h}$. The cells were washed with phosphate-buffered saline and fixed with formaldehyde. The fixed cells were stained with oil Red $\mathrm{O}$ dye, for $30 \mathrm{~min}$ at $37^{\circ} \mathrm{C}$. The status of intracellular lipid accumulation was detected by microscopy (Olympus CKX41; Olympus, Tokyo, Japan), in order to assess the formation of macrophage foam cells.

Western blotting. The J774A.1 cells were plated in 6-well plates and grown to 50-60\% confluency. Following stimulation with ox-LDL (or/and simvastatin at 0.5 and $1.0 \mu \mathrm{M}$ ), the cells were harvested and lysed with RIPA lysis buffer on ice. The cell lysates were centrifuged at $13,000 \mathrm{~g}$ for $30 \mathrm{~min}$ at $4^{\circ} \mathrm{C}$. The protein concentrations of the supernatants were determined using the BCA kit. Equal quantities of protein $(30 \mu \mathrm{g})$ from the homogenized samples were separated by $15 \%$ SDS-PAGE, and transferred to polyvinylidene fluoride membranes (Millipore, Boston, MA, USA). The membranes were blocked with tris-buffered saline-Tween ${ }^{\circledR}$ (TBST), containing 5\% non-fat milk powder, for $2 \mathrm{~h}$ at room temperature. The membranes were then incubated with polyclonal rabbit anti-microtubule-associated protein 1 light chain 3(LC3; $1: 1,000)$ and polyclonal rabbit anti-Beclin1 $(1: 1,000)$ primary antibodies at $4^{\circ} \mathrm{C}$ overnight, washed with TBST, and incubated for $1 \mathrm{~h}$ with HRP-conjugated goat anti-rabbit antibody (1:10,000 dilution), at room temperature. $\beta$-actin was used as a control. Immunoreactive bands were visualized using the ECL detection system and images were captured using an automatic digital gel image analysis system (4500SF; Tanon Science \& Technology Co., Ltd., Shanghai, China).

Confocal laser scanning microscopy. Green fluorescent protein (GFP)-LC3 plasmids were previously prepared in our labora- tory (15) and were transfected into the J774A.1 cells, using Lipofectamine ${ }^{\circledR} 2000$ according to the manufacturer's instructions. The cells were treated with ox-LDL (and/or simvastatin) $24 \mathrm{~h}$ post-transfection, and incubated for a further $24 \mathrm{~h}$. All GFP-LC3 plasmid-transfected cells with or without ox-LDL (and/or simvastatin) treatment were then fixed using Immunol Staining Fix Solution (Beyotime Institute of Biotechnology, Haimen, China) and GFP-LC3 fluorescence was detected by confocal laser scanning microscopy (Leica TCS SP5; Leica, Mannheim, Germany).

Measurement of intracellular cholesterol using the CHOD-PAP method. Following stimulation with ox-LDL (or/and simvastatin), the cells were harvested and sonicated on ice for a total of $5 \mathrm{sec}$, with $15 \mathrm{sec}$ pauses between bursts using a sonicator (Ningbo Scientz Biotechnology Co., Ltd., Ningbo,China). Following sonication for $10 \mathrm{~min}$, the cells were centrifuged at $8,000 \mathrm{~g}$ for $10 \mathrm{~min}$ at $4^{\circ} \mathrm{C}$. The supernatants were then used for measurement of intracellular cholesterol. Total cholesterol levels in the cells were determined by the CHOD-PAP method, using the Total Cholesterol Detection kit (Nanjing Jiancheng Biotechnology Institute Co., Ltd.), according to the manufacturer's instructions.

Statistical analysis. The data are represented as the mean \pm standard deviation, and were statistically analyzed using SPSS 13.0 software (SPSS Inc., Chicago, IL, USA). Statistical analysis was performed using Student's t-test to compare the differences between two groups. $\mathrm{P}<0.05$ was considered to indicate a statistically significant difference.

\section{Results}

Ox-LDL induces lipid accumulation in macrophages. To investigate the possible roles of simvastatin on ox-LDL-induced macrophage autophagy and lipid accumulation, a macrophage foam cell model was generated. Groups A, B, C and D of the J774A. 1 cells were treated with $0,25,50$ and $100 \mu \mathrm{g} / \mathrm{ml}$ ox-LDL respectively, for $24 \mathrm{~h}$, and the status of lipid accumulation was determined by oil red $\mathrm{O}$ staining. No obvious lipid accumulation was observed in the control group (Fig. 1A). Conversely, lipid accumulation was observed in all of the groups treated with ox-LDL (Fig. 1B-D). Lipid accumulation was more visible in groups $\mathrm{C}$ and $\mathrm{D}$, as compared with group B, implying the existence of a dose-dependent effect. All of the cells treated with ox-LDL had similar morphological characteristics to foam cells, suggesting the successful establishment of a macrophage foam cell model. Thereafter, $50 \mu \mathrm{g} / \mathrm{ml}$ ox-LDL was selected to treat cells in further experiments.

Ox-LDL induces the transformation of LC3 I to LC3 II in macrophages. Ox-LDLs have previously been shown to trigger autophagy in endothelial cells $(8,9,16)$. The present study examined whether ox-LDL induced autophagy in macrophages. Ox-LDL $(50 \mu \mathrm{g} / \mathrm{ml})$ induced autophagy in the J774A.1 macrophage cell line by converting LC3 I to LC3 II, which is a well-known autophagy marker. The transformation of LC3 I to LC3 II increased gradually, in a time-dependent manner following treatment with $50 \mu \mathrm{g} / \mathrm{ml}$ ox-LDL, as compared with the control group (Fig. 2). 

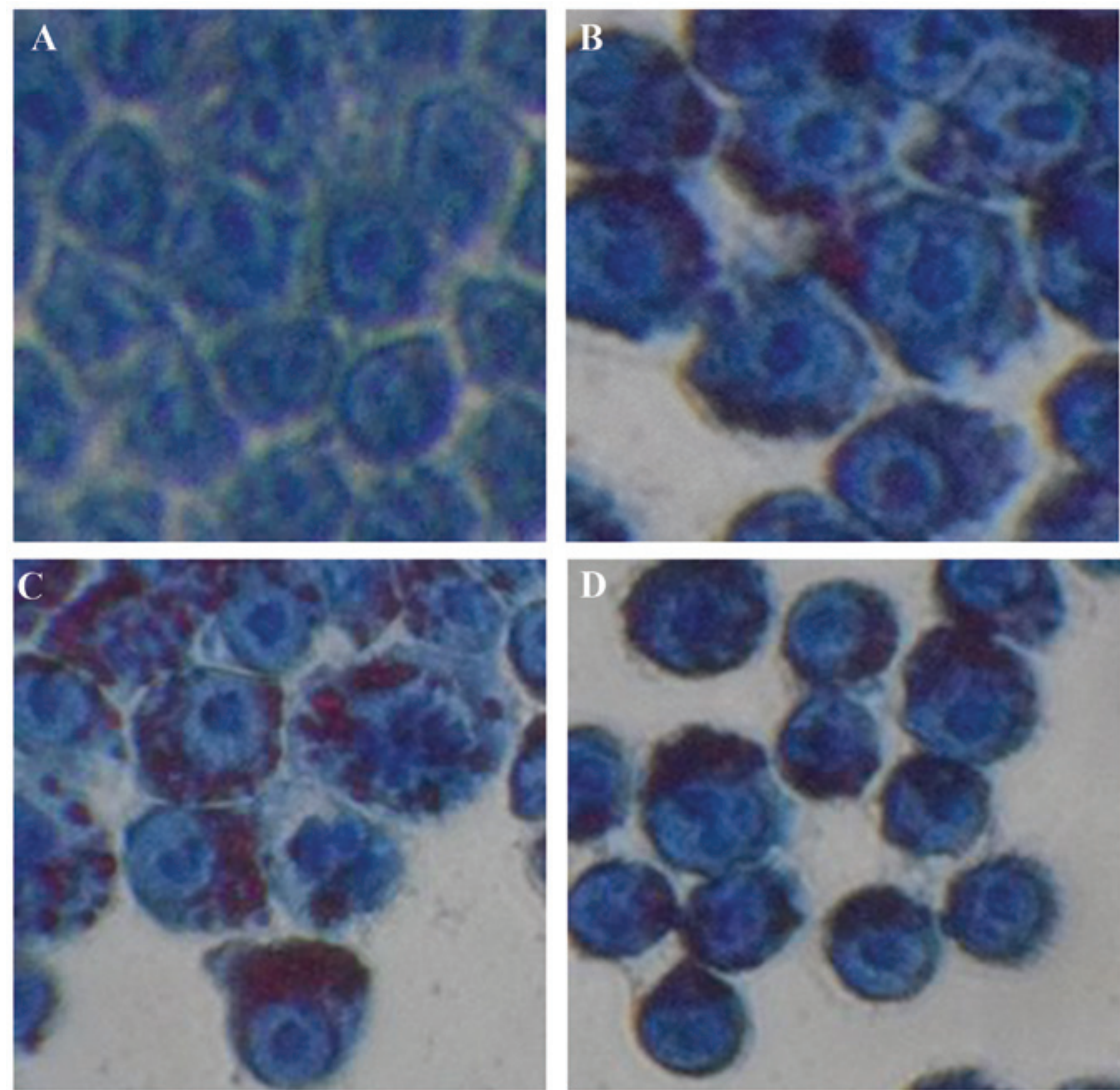

Figure 1. ox-LDL promotes lipid accumulation in the J774A.1 macrophage cell line. (A) 0 (untreated control group), (B) 25 , (C) 50 and (D) $100 \mu \mathrm{g} / \mathrm{ml}$ ox-LDL was added to the supernatants of the J774A.1 cells. Following a $24 \mathrm{~h}$ incubation, lipid accumulation was detected by oil red $\mathrm{O}$ staining. Magnification, $\mathrm{x} 400$. ox-LDL, Oxidized low-density lipoprotein.

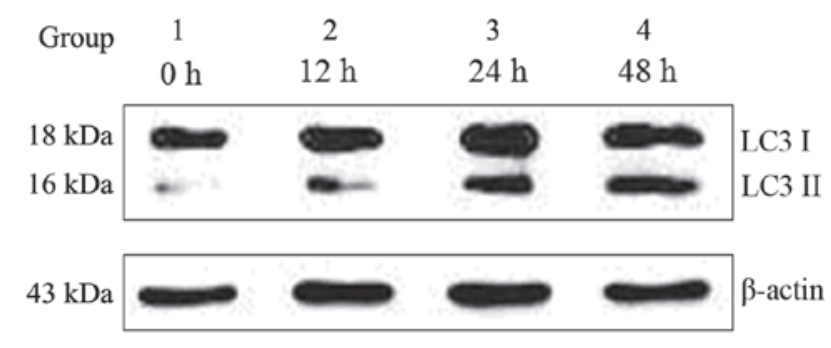

Figure 2. ox-LDL induces the conversion of LC3I to LC3 II, in the J774A.1 macrophage cell line. The cells were treated with $50 \mu \mathrm{g} / \mathrm{ml}$ ox-LDL for 0,12 , 24 or $48 \mathrm{~h}$, and the transformation of LC3 I to LC3 II was detected by western blotting. ox-LDL, oxidized low-density lipoprotein; LC3, microtubule-associated protein 1 light chain 3 .

Simvastatin enhances ox-LDL-induced macrophage autophagy. Simvastatin was previously shown to enhance autophagy in osteoblastic cells and coronary arterial myocytes $(14,17)$. The present study determined whether simvastatin elevated ox-LDL-induced autophagy in macrophages. Concordant with the results presented in Fig. 2, the protein expression levels of the autophagy marker LC3 II were induced in group 2, following stimulation with $50 \mu \mathrm{g} / \mathrm{ml}$ ox-LDL for $24 \mathrm{~h}$ (Fig. 3). Notably, treatment with simvastatin markedly promoted the conversion of LC3 I to LC3 II (group 4 and 5, as compared with group 2). Treatment with $1.0 \mu \mathrm{M}$ simvastatin enhanced ox-LDL-induced LC3 II expression more than treatment with $0.5 \mu \mathrm{M}$ simvastatin, suggesting that simvastatin affects ox-LDL-induced autophagy in a dose-dependent manner (Fig. 3). Simvastatin

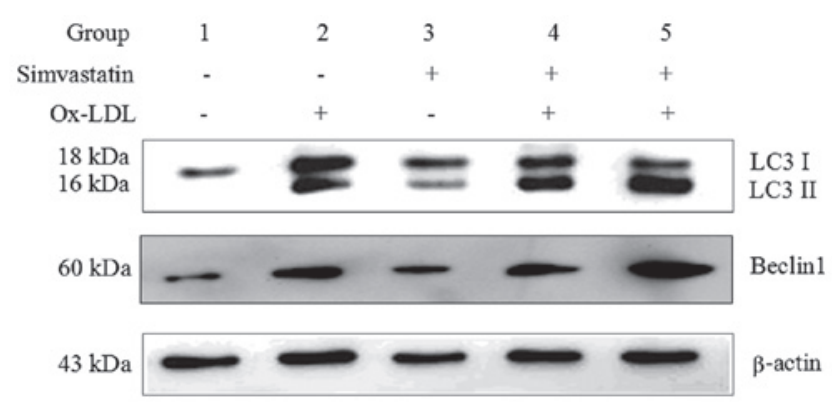

Figure 3. Simvastatin enhances ox-LDL-induced macrophage autophagy. The J774A.1 macrophage cells were treated with $50 \mu \mathrm{g} / \mathrm{ml}$ ox-LDL (group 2), $1.0 \mu \mathrm{M}$ simvastatin (group 3), $50 \mu \mathrm{g} / \mathrm{ml}$ ox-LDL plus $0.5 \mu \mathrm{M}$ simvastatin (group 4), or $50 \mu \mathrm{g} / \mathrm{ml}$ ox-LDL plus $1.0 \mu \mathrm{M}$ simvastatin (group 5). Following a $24 \mathrm{~h}$ incubation, the cells were harvested, and a western blot analysis was performed. Group 1 was the untreated control. ox-LDL, oxidized low-density lipoprotein; LC3, microtubule-associated protein 1 light chain 3.

$(0.5$ and $1.0 \mu \mathrm{M})$ also increased the expression levels of another ox-LDL-induced autophagy marker, Beclin1 (Fig. 3).

The GFP-LC3 plasmid was used to detect autophagosome formation. Stimulation with ox-LDL led to the redistribution of GFP-LC3, from diffusion distribution to puncta in the J774A.1 cells, as determined by confocal laser scanning microscopy (Fig. 4A and B). Treatment with simvastatin ( 0.5 and $1.0 \mu \mathrm{M}$ ) alone resulted in the formation of GFP-LC3 fluorescent puncta in the J774A.1 cells (Fig. 4C and D). Furthermore, treatment with simvastatin markedly promoted the ox-LDL-induced 

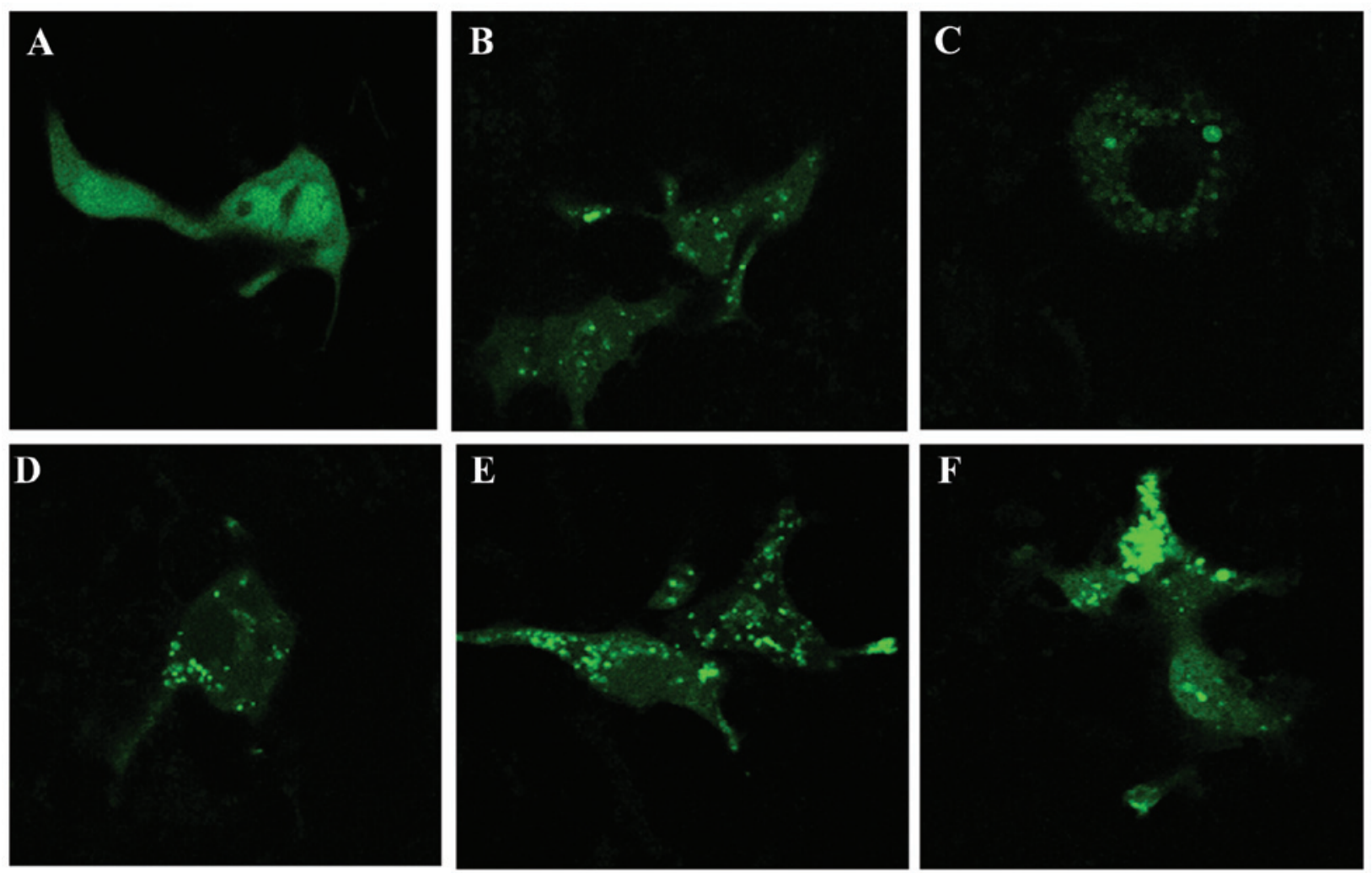

Figure 4. Simvastatin promotes ox-LDL-induced formation of GFP-LC3 fluorescent puncta. J774A.1 macrophage cells were transfected with plasmids containing GFP-LC3, following a $24 \mathrm{~h}$ incubation the cells were either (A) left untreated (control), or treated with (B) $50 \mu \mathrm{g} / \mathrm{ml}$ ox-LDL, (C) simvastatin (0.5 $\mu \mathrm{M}$ ), (D) $1.0 \mu \mathrm{M}$ simvastatin, (E) $50 \mu \mathrm{g} / \mathrm{ml}$ ox-LDL plus $0.5 \mu \mathrm{M}$ simvastatin or (F) $50 \mu \mathrm{g} / \mathrm{ml}$ ox-LDL plus $1.0 \mu \mathrm{M}$ simvastatin for a further $24 \mathrm{~h}$. GFP-LC3 fluorescent puncta were detected by confocal laser scanning microscopy. ox-LDL, oxidized low-density lipoprotein; GFP, green fluorescent protein; LC3, microtubule-associated protein 1 light chain 3.
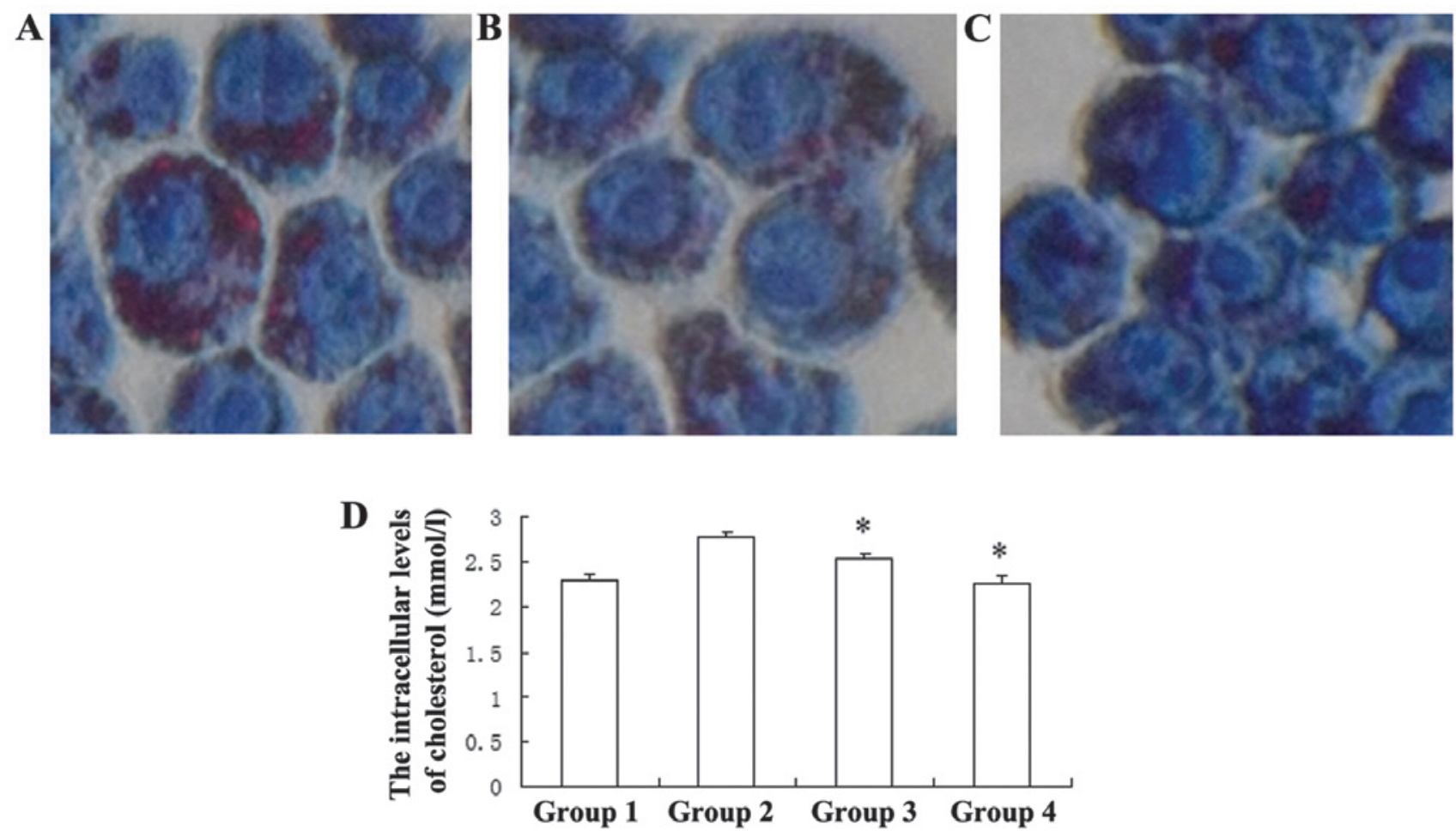

Figure 5. Simvastatin inhibits ox-LDL-induced cholesterol accumulation in macrophages. J774A.1 macrophage cells were treated with (A) $50 \mu \mathrm{g} / \mathrm{ml}$ ox-LDL, $50 \mu \mathrm{g} / \mathrm{ml}$ ox-LDL plus (B) 0.5 or (C) $1.0 \mu \mathrm{M}$ simvastatin for $24 \mathrm{~h}$. The intracellular lipid droplets were detected by red oil O staining. (D) Intracellular total cholesterol levels were assessed using the CHOD-PAP method after the J774A.1 cells were treated with $50 \mu \mathrm{g} / \mathrm{ml}$ ox-LDL (group 2), $50 \mu \mathrm{g} / \mathrm{ml}$ ox-LDL plus 0.5 (group 3) or $1.0 \mu \mathrm{M}$ simvastatin (group 4) for $24 \mathrm{~h}$ respectively. Group 1 refers to the untreated control. The data are represented as the mean \pm standard deviation. ${ }^{*} \mathrm{P}<0.01$, ox-LDL-treated group vs. ox-LDL plus simvastatin-treated group. ox-LDL, oxidized low-density lipoprotein. 
formation of GFP-LC3 puncta (Fig. 4E and F). Treatment with $1.0 \mu \mathrm{M}$ simvastatin enhanced the formation of ox-LDL-induced GFP-LC3 puncta more so than $0.5 \mu \mathrm{M}$ simvastatin (Fig. 4E and F). These results indicate that simvastatin may enhance ox-LDL-induced macrophage autophagy.

Simvastatin inhibits ox-LDL-induced cholesterol accumulation inmacrophages. As aHMG-CoA reductase inhibitor, simvastatin is a well-known drug used to ameliorate atherosclerotic diseases, by lowering plasma cholesterol. The present study determined whether simvastatin may inhibit ox-LDL-induced cholesterol accumulation in J774A.1 cells. The number of lipid droplets, the existing form of cholesterol in cells, decreased following incubation with simvastatin (Fig. 5A-C). The CHOD-PAP assay indicated that treatment with 0.5 and $1.0 \mu \mathrm{M}$ simvastatin led to a significant reduction in the intracellular total cholesterol levels, from $2.774 \pm 0.059 \mathrm{mmol} / 1$, to $2.53 \pm 0.058 \mathrm{mmol} / 1$ and $2.27 \pm 0.085 \mathrm{mmol} / 1$, respectively (Fig. 5D). These results suggest that simvastatin may inhibit ox-LDL-induced cholesterol accumulation in macrophages.

\section{Discussion}

Ox-LDL has a pivotal role in atherosclerosis initiation and progression. Ox-LDL may be recognized and engulfed by macrophages through scavenger receptors, leading to the formation of foam cells, which are the prominent feature of atherosclerotic lesions $(18,19)$. As predicted, the results of the present study demonstrated that ox-LDL was capable of inducing lipid accumulation in J74A A.1 cells, indicating the formation of foam cells. Previous studies have shown that ox-LDL may induce autophagy in endothelial cells $(8,9,16,20)$. However, it is relatively difficult to observe autophagy in macrophages, since they are phagocytic and the cytoplasmic vacuoles render it difficult to distinguish between autophagocytosis and heterophagocytosis (4). Ouimet and Marcel (21) reported that acetylated-LDL elevated macrophage intracellular cholesterol levels, and were delivered to the lysosomes by autophagy (21). Concordant with these results, the present study demonstrated that ox-LDL induced autophagy in macrophages, by increasing the expression of LC3 II and Beclin1, the classic autophagy markers.

As a powerful lipid-lowering drug, simvastatin is widely used for the treatment of atherosclerosis. Accumulating evidence has shown that simvastatin also has various other effects. The present study reported that, besides decreasing ox-LDL-induced cholesterol accumulation, simvastatin intensified ox-LDL-induced macrophage autophagy. However, it remains noteworthy that drugs may directly induce macrophage autophagy in atherosclerosis, and certain drug-induced macrophage autophagy promotes a stable plaque phenotype (22). The results of the present study showed that simvastatin may directly induce macrophage autophagy, albeit only marginally. Whether this effect may have a role in plaque progression requires further investigation.

In mammalian cells there are two types of autophagy: mTOR-dependent and mTOR-independent autophagy (22). Wei et al (14) demonstrated that simvastatin induced autophagy through inhibition of Racl-mTOR signaling in coronary arterial myocytes. However, it remains unknown whether simvastatin promotes ox-LDL-induced macrophage autophagy in the same way. Further studies are required to explore whether ox-LDL-induced macrophage autophagy is mTOR-dependent or not. In addition, the results of the present study were obtained from in vitro experiments. An animal model of atherosclerosis may be useful to confirm these findings.

In conclusion, the results of the present study suggest that ox-LDL is capable of inducing autophagy in macrophages, and simvastatin may enhance ox-LDL-induced macrophage autophagy and attenuate lipid accumulation. These results imply that macrophage autophagy in atherosclerosis may be the potential target of simvastatin for plaque stabilization.

\section{Acknowledgements}

The present study was supported by grants from the National Natural Science Foundation of China Grants (grant no. 30972791, to Baojun Huang) and the Research Fund of Anhui Medical University (grant no. 0116025101, to Songcheng Ying).

\section{References}

1. Legein B,Temmerman L, Biessen EA and Lutgens E: Inflammation and immune system interactions in atherosclerosis. Cell Mol Life Sci 70: 3847-3869, 2013

2. Cybulsky MI, Won D and Haidari M: Leukocyte recruitment to atherosclerotic lesions. Can J Cardiol 20: 24B-28B, 2004.

3. Moore KJ, Sheedy FJ and Fisher EA: Macrophages in atherosclerosis: a dynamic balance. Nat Rev Immunol 13: 709-721, 2013.

4. Martinet W and De Meyer GR: Autophagy in atherosclerosis. Curr Atheroscler Rep 10: 216-223, 2008.

5. Martinet W and De Meyer GR: Autophagy in atherosclerosis: a cell survival and death phenomenon with therapeutic potential. Circ Res 104: 304-317, 2009.

6. Schrijvers DM, De Meyer GR and Martinet W: Autophagy in atherosclerosis: a potential drug target for plaque stabilization. Arterioscler Thromb Vasc Biol 31: 2787-2791, 2011.

7. Liao X, Sluimer JC, Wang Y, Subramanian M, Brown K, Pattison JS, Robbins J, Martinez J and Tabas I: Macrophage autophagy plays a protective role in advanced atherosclerosis. Cell Metab 15: 545-553, 2012.

8. Zhang YL, Cao YJ, Zhang X, Liu HH, Tong T, Xiao GD, Yang YP and Liu CF: The autophagy-lysosome pathway: a novel mechanism involved in the processing of oxidized LDL in human vascular endothelial cells. Biochem Biophys Res Commun 394: 377-382, 2010.

9. Muller C, Salvayre R, Nègre-Salvayre A and Vindis C: HDLs inhibit endoplasmic reticulum stress and autophagic response induced by oxidized LDLs. Cell Death Differ 18: 817-828, 2011.

10. Almuti K, Rimawi R, Spevack D and Ostfeld RJ: Effects of statins beyond lipid lowering: potential for clinical benefits. Int J Cardiol 109: 7-15, 2006.

11. Bu DX, Griffin G and Lichtman AH: Mechanisms for the anti-inflammatory effects of statins. Curr Opin Lipidol 22: 165-170, 2011.

12. Wierzbicki AS, Poston R and Ferro A: The lipid and non-lipid effects of statins. Pharmacol Ther 99: 95-112, 2003.

13. Marzilli M: Pleiotropic effects of statins: evidence for benefits beyond LDL-cholesterol lowering. Am J Cardiovasc Drugs 10 (Suppl 1): 3-9, 2010.

14. Wei YM, Li X, Xu M, Abais JM, Chen Y, Riebling CR, Boini KM, Li PL and Zhang Y: Enhancement of autophagy by simvastatin through inhibition of Rac1-mTOR signaling pathway in coronary arterial myocytes. Cell Physiol Biochem 31: 925-937, 2013.

15. Zhang M, Huang BJ, Hu MC, Chen YW, Song W, Huang DK, Li Q and $\mathrm{Hu}$ CS: Lipopolysaccharide induces formation of autophagyrelated LC3B-GFP fluorescent aggregates in the RAW264.7 macropahge cells. J Anhui Medical Uni 47: 357-361, 2012 (In Chinese). 
16. Nowicki M, Zabirnyk O, Duerrschmidt N, Borlak J and Spanel-Borowski K: No upregulation of lectin-like oxidized low-density lipoprotein receptor-1 in serum-deprived EA.hy926 endothelial cells under oxLDL exposure, but increase in autophagy. Eur J Cell Biol 86: 605-616, 2007.

17. Lai EH, Hong CY, Kok SH, Hou KL, Chao LH, Lin LD, Chen MH, Wu PH and Lin SK: Simvastatin alleviates the progression of periapical lesions by modulating autophagy and apoptosis in osteoblasts. J Endod 38: 757-763, 2012.

18. Maiolino G, Rossitto G, Caielli P,Bisogni V, Rossi GP and Calò LA The role of oxidized low-density lipoproteins in atherosclerosis: the myths and the facts. Mediators Inflamm 2013: 714653, 2013.
19. Mitra S, Goyal T and Mehta JL: Oxidized LDL, LOX-1 and atherosclerosis. Cardiovasc Drugs Ther 25: 419-429, 2011.

20. Ding Z, Wang X, Khaidakov M, Liu S, Dai Y and Mehta JL: Degradation of heparan sulfate proteoglycans enhances oxidized-LDL-mediated autophagy and apoptosis in human endothelial cells. Biochem Biophys Res Commun 426: 106-111, 2012.

21. Ouimet M and Marcel YL: Regulation of lipid droplet cholesterol efflux from macrophage foam cells. Arterioscler Thromb Vasc Biol 32: 575-581, 2012.

22. Martinet W, De Meyer I, Verheye S, Schrijvers DM, Timmermans JP and De Meyer GR: Drug-induced macrophage autophagy in atherosclerosis: for better or worse? Basic Res Cardiol 108: 321, 2013. 